\title{
A Passive Microwave Retrieval Algorithm with Minimal View-Angle Bias: Application to the TEMPEST-D CubeSat Mission
}

\author{
Richard M. Schulte, Christian D. Kummerow, and Wesley BerG \\ Department of Atmospheric Science, Colorado State University, Fort Collins, Colorado \\ STEVEN C. REISING \\ Department of Electrical and Computer Engineering, Colorado State University, Fort Collins, Colorado \\ Shannon T. Brown, Todd C. Gaier, Boon H. Lim, and Sharmila Padmanabhan \\ Jet Propulsion Laboratory, California Institute of Technology, Pasadena, California
}

(Manuscript received 26 September 2019, in final form 27 November 2019)

\begin{abstract}
The rapid development of miniaturized satellite instrument technology has created a new opportunity to deploy constellations of passive microwave (PMW) radiometers to permit retrievals of the same Earth scene with very high temporal resolution to monitor cloud evolution and processes. For such a concept to be feasible, it must be shown that it is possible to distinguish actual changes in the atmospheric state from the variability induced by making observations at different Earth incidence angles (EIAs). To this end, we present a flexible and physical optimal estimation-based algorithm designed to retrieve profiles of atmospheric water vapor, cloud liquid water path, and cloud ice water path from cross-track PMW sounders. The algorithm is able to explicitly account for the dependence of forward model errors on EIA and atmospheric regime. When the algorithm is applied to data from the Temporal Experiment for Storms and Tropical Systems Technology Demonstration (TEMPEST-D) CubeSat mission, its retrieved products are generally in agreement with those obtained from the similar but larger Microwave Humidity Sounder instrument. More importantly, when forward model brightness temperature offsets and assumed error covariances are allowed to change with EIA and sea surface temperature, view-angle-related biases are greatly reduced. This finding is confirmed in two ways: through a comparison with reanalysis data and by making use of brief periods in early 2019 during which the TEMPEST-D spacecraft was rotated such that its scan pattern was along track, allowing dozens of separate observations of any given atmospheric feature along the satellite's ground track.
\end{abstract}

\section{Introduction}

Satellite-based passive microwave (PMW) radiometers have been used for several decades to measure atmospheric water vapor and bulk cloud properties such as total liquid water path and total ice water path (e.g., Wilheit and Chang 1980; Greenwald et al. 1993; Wentz 1997; Boukabara et al. 2010). PMW instruments also provide some of the most important observations for operational data assimilation (Geer et al. 2017). Recently, rapid advances in miniaturized satellite instrument technology have opened the door to making

Corresponding author: Richard Schulte,rschulte@atmos. colostate.edu
PMW measurements from U-class satellites known as CubeSats. These much smaller satellite platforms could allow a larger number of satellites to be launched and many more PMW observations to be made, combining high temporal resolution that is unachievable from existing PMW satellite instruments with sensitivity to changes below the cloud top that is missing from geostationary visible and infrared measurements. This would provide both operational forecasters and data assimilation systems with additional useful information.

Moreover, while traditional PMW missions have focused on global mapping, cost-efficient PMW CubeSat missions facilitate the design of process-oriented studies that make use of constellations or "trains" of satellites to make repeated observations of atmospheric phenomena 


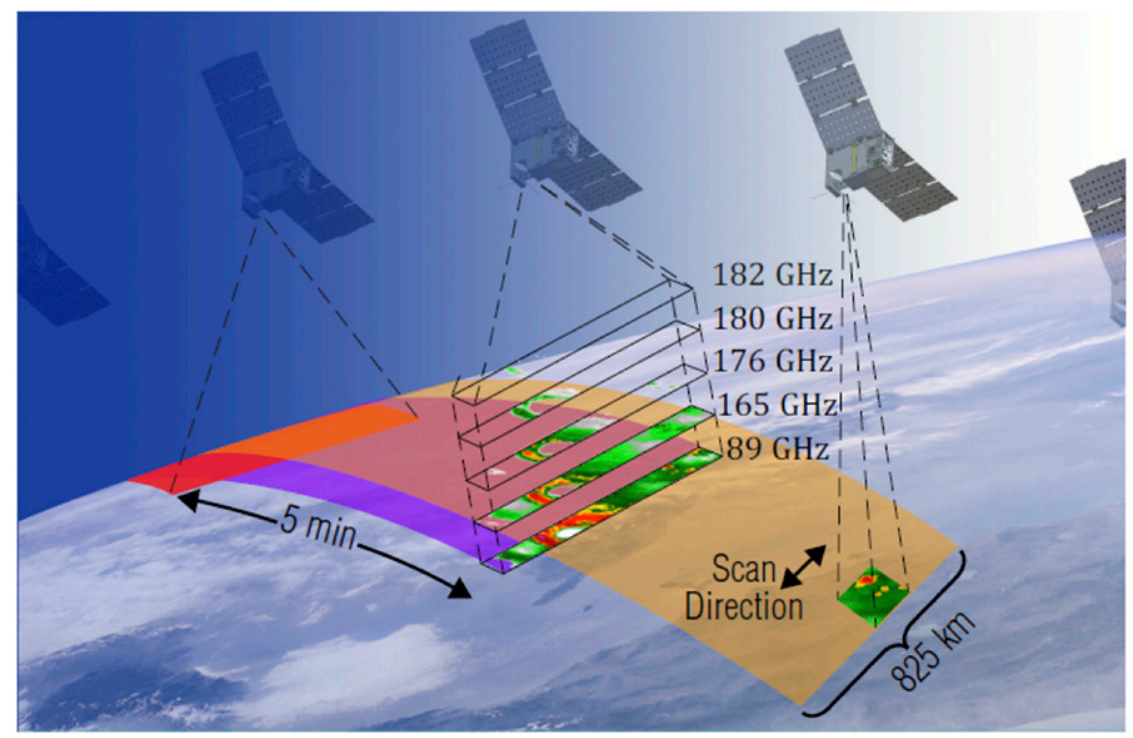

FIG. 1. Conceptual illustration of a TEMPEST train of CubeSats.

that occur on time scales of a few minutes to an hour or so (Ma et al. 2017). The proposed Temporal Experiment for Storms and Tropical Systems (TEMPEST) mission would consist of a cluster of 6-8 CubeSats carrying identical PMW radiometers in the same orbital plane. They would be separated by only a few minutes and thus could make repeated measurements of the same convective cells in order to better understand the evolution of these storm systems and the forcing and feedbacks between convection and midtropospheric water vapor. An illustration of the concept is presented in Fig. 1.

To realize these potential applications of PMW CubeSat missions, it is necessary to demonstrate that they can provide science-quality measurements that are on par with those provided by similar instruments on larger satellites. For potential constellation "trains" designed to study cloud processes, one must also determine whether actual changes to the atmospheric state that occur over only a few minutes can be distinguished from the uncertainties involved in making measurements from two different satellites with different slant paths through the atmosphere (as illustrated in Fig. 1, even two satellites in the same orbital plane will view the same spot on Earth from slightly different angles because of the rotation of Earth during the time between successive observations).

With the launch of the TEMPEST Demonstration (TEMPEST-D) CubeSat, a technology demonstration mission currently in orbit (Reising et al. 2018), it is now possible to begin addressing some of these questions directly. In particular, using yaw maneuvers performed by the TEMPEST-D spacecraft, we can assess view-angle biases in a novel way, by comparing retrievals made by the same instrument at nearly the same time and over nearly the same area, but from different view angles. In this paper we apply the CSU 1DVAR retrieval algorithm (Schulte and Kummerow 2019; hereinafter SK19) to TEMPEST-D observations to retrieve total precipitable water (TPW), cloud liquid water path (LWP), and cloud ice water path (IWP) to answer two key questions:

1) Do the TEMPEST-D measurements yield TPW, LWP, and IWP estimates that are consistent with those from the Microwave Humidity Sounder (MHS) class of PMW radiometers?

2) Do the TEMPEST-D estimates exhibit any bias as a function of view angle, and are the view-angle-related uncertainties small enough that real changes in the atmospheric state can be distinguished from measurement uncertainty?

In section 2 we further describe TEMPEST-D as well as our other data sources. Section 3 provides a brief overview of the CSU 1DVAR algorithm and describes how we construct error covariance matrices and forward model brightness temperature offsets. In section 4 we answer the key questions outlined above, and in section 5 we discuss implications for future satellite missions.

\section{Data}

The TEMPEST-D satellite was launched on 21 May 2018 on a commercial resupply mission to the International Space Station and was successfully deployed into an orbit with an altitude of $400 \mathrm{~km}$ and inclination of $51.6^{\circ}$ on 
TABLE 1. Selected sensor specifications for TEMPEST-D and MHS.

\begin{tabular}{lcc}
\hline & TEMPEST-D & MHS \\
\hline Channel frequency $(\mathrm{GHz})$ & $87,164,174,178$, and 181 & $89,157,183 \pm 1,183 \pm 3$, and 190 \\
Mass & $3.8 \mathrm{~kg}$ & $63 \mathrm{~kg}$ \\
Power & $6.5 \mathrm{~W}$ & $74 \mathrm{~W}$ \\
Altitude & $400 \mathrm{~km}$ & $820 \mathrm{~km}$ \\
Resolution at nadir & $12.5 \mathrm{~km}(25 \mathrm{~km}$ at $87 \mathrm{GHz})$ & $15.9 \mathrm{~km}$ \\
NEDT (K) & $0.2,0.3,0.4,0.4$, and 0.7 & $0.22,0.34,0.46,0.40$, and 0.51 \\
Integration time & $5 \mathrm{~ms}$ & $18.5 \mathrm{~ms}$ \\
\hline
\end{tabular}

13 July 2018. The $6 \mathrm{U}(34 \mathrm{~cm} \times 20 \mathrm{~cm} \times 10 \mathrm{~cm})$ CubeSat carries as its main payload a five-channel passive microwave radiometer operating in bands centered at $87,164,174,178$, and $181 \mathrm{GHz}$. The $87-\mathrm{GHz}$ channel has quasi-vertical polarization (vertical polarization at nadir), whereas the other channels have quasi-horizontal polarization. Additional technical specifications are provided in Table 1, along with comparable values for the MHS radiometer, which operates at a similar set of frequencies on board the MetOp-A, MetOp-B, MetOp-C, and NOAA-19 spacecraft. Note that the mass and power of TEMPEST-D are far lower than those of operational PMW sensors and also that TEMPEST-D has noise characteristics [as measured by noise equivalent differential temperature (NEDT)] that are similar to those of MHS despite a significantly shorter integration time. For the same integration time, TEMPEST-D has lower noise than MHS.

Because of the roughly 1-yr period of time during which TEMPEST-D has been making measurements, as well as limitations in transmitting the collected data from the spacecraft to the ground, only limited data are available with which to evaluate the TEMPEST-D measurements and retrieval algorithm. This makes a comprehensive validation study difficult but does not prohibit our objectives-namely, demonstrating general agreement with MHS observations and examining viewangle-related biases. In this study, we use one week of continuous data from 8 to 14 December 2018 to meet the first objective and to calculate forward model bias corrections.

For the second objective, we make use of special periods during which the TEMPEST-D spacecraft was intentionally yawed by $92^{\circ}$, thus providing along-track scanning during portions of the descending node of each orbit and nearly along-track scanning during the other periods (the exact degree to which the scans overlap is dependent both on the latitude and the direction of spacecraft motion). This dataset is to our knowledge the first of its kind from a spaceborne sensor and provides multiple observations of certain points on Earth from wide-ranging view angles by the same instrument and at nearly the same time. All told, we have collected about $73 \mathrm{~h}$ (or about 11.5 million retrieved pixels) of alongtrack observations, during January and April 2019.

Ancillary data (surface wind speeds, surface pressures, temperature profiles, and sea surface temperatures) used by the CSU 1DVAR retrieval algorithm are taken from the Goddard Earth Observing System Model, version 5 (GEOS-5; Molod et al. 2012). The a priori water vapor profile used by the algorithm also comes from GEOS-5. GEOS-5 data are used (unlike reanalysis data as in SK19) to be able to run the retrieval in near-real time. We examine coincident overpasses between TEMPEST$\mathrm{D}$ and MHS, and compare the values retrieved by the TEMPEST-D algorithm with the corresponding MHS values from the Microwave Integrated Retrieval System (MiRS; Boukabara et al. 2011) and with CSU 1DVAR retrievals run on the MHS data. MiRS Orbital Level-2 output is obtained from the NOAA Comprehensive Large Array-Data Stewardship System (CLASS), and we use version 11.2 of the algorithm. All satellites carrying an MHS sensor also have an Advanced Microwave Sounding Unit-A (AMSU-A), which has 15 channels at frequencies ranging from 23.8 to $89.0 \mathrm{GHz}$ and is primarily used for temperature sounding. Note that radiances from this instrument are taken into account in the MiRS algorithm but not in the CSU 1DVAR algorithm.

\section{Methods \\ a. CSU 1DVAR}

The algorithm with which we retrieve TPW, LWP, and IWP from TEMPEST-D brightness temperatures $T_{b}$ is an extension of the optimal estimation algorithm developed in Duncan and Kummerow (2016). In brief, the 1DVAR or optimal estimation technique is an inverse method based on Bayes's theorem, that is,

$$
P(\mathbf{x} \mid \mathbf{y})=\frac{P(\mathbf{y} \mid \mathbf{x}) P(\mathbf{x})}{P(\mathbf{y})} .
$$

In this case, $\mathbf{y}$ is the measurement vector containing the observed $T_{b}$ and $\mathbf{x}$ is the state vector consisting of the 
atmospheric properties to be estimated (LWP, IWP, and the three leading principal components of the water vapor profile). Given radiometer observations $\mathbf{y}$, we wish to find the value of $\mathbf{x}$ for which the posterior probability $P(\mathbf{x} \mid \mathbf{y})$ is maximized. If we consider $P(\mathbf{y})$ to be a normalizing factor, then this amounts to maximizing the product of the a priori probability of a given state $P(\mathbf{x})$ with the probability of measuring the set of $T_{b}$ if $\mathbf{x}$ were indeed the state of the atmosphere $P(\mathbf{y} \mid \mathbf{x})$. The relationship between $\mathbf{x}$ and $\mathbf{y}$ can be generalized by

$$
\mathbf{y}=f(\mathbf{x}, \mathbf{b})+\varepsilon,
$$

where $f$ is a forward model based on radiative transfer theory. The forward model requires additional information besides the parameters given in $\mathbf{x}$ to be able to calculate simulated $T_{b}$ (such as the temperature profile, the surface wind speed, the cloud structure, etc.), and these various assumptions are represented by the vector $\mathbf{b} ; \boldsymbol{\varepsilon}$ is an error term containing uncertainties due to sensor noise, errors in the forward model, and uncertainties in the ancillary parameters b. In theory, the forward modeled brightness temperatures $f(\mathbf{x}, \mathbf{y})$ should agree with the satellite measurements $\mathbf{y}$ within the model and sensor error estimates given by $\boldsymbol{\varepsilon}$. The aim of the 1DVAR algorithm is to find the most likely state vector $\mathbf{x}$, given measurements $\mathbf{y}$, prior knowledge about the state of the atmosphere, and proper error estimates.

It can be shown (Rodgers 2000) that, under the assumption of Gaussian errors in the assumed a priori state and in observed and forward modeled $T_{b}$, maximizing the numerator on the right-hand side of Eq. (1) is equivalent to minimizing the cost function $\Phi$ :

$$
\begin{aligned}
\Phi= & \left(\mathbf{x}-\mathbf{x}_{a}\right)^{\mathrm{T}} \mathbf{S}_{a}^{-1}\left(\mathbf{x}-\mathbf{x}_{a}\right) \\
& +[\mathbf{y}-f(\mathbf{x}, \mathbf{b})]^{\mathrm{T}} \mathbf{S}_{y}^{-1}[\mathbf{y}-f(\mathbf{x}, \mathbf{b})] .
\end{aligned}
$$

The first term in $\Phi$ indicates how far a potential solution is from the assumed a priori state $\mathbf{x}_{a}$. This difference is weighted by the assumed errors in the a priori parameters and their covariances, as described by the a priori covariance matrix $\mathbf{S}_{a}$. The second term is a measure of the difference between observed and forward modeled $T_{b}$, and it contains a second error covariance matrix, the $\mathbf{S}_{y}$ matrix, which describes the uncertainties in both the observations and the forward model. The Gauss-Newton method is used to iteratively solve for the value of $\mathbf{x}$ at which the gradient of the cost function $\nabla_{x} \Phi$ is equal to zero.

We use the same forward model as in SK19, the key components being version 5.3 of the Monochromatic Radiative Transfer Model (MonoRTM; Clough et al. 2005) for calculating absorption coefficients and the FASTEM6 model of ocean surface emissivity (Kazumori and English 2015). We also make the same assumptions as in SK19 about cloud composition and height. To summarize, cloud water is distributed evenly between the pressure levels of 800 and $925 \mathrm{hPa}$, with an assumed monodisperse drop size distribution (DSD) of spherical cloud droplets with radii of $12 \mu \mathrm{m}$. Ice particles are likewise distributed evenly between 300 and $400 \mathrm{hPa}$, with a parameterization of the ice particle size distribution that comes from Field et al. (2007), and scattering calculated according to a database of single-scattering properties at microwave frequencies for various ice crystal habits (Liu 2008) as well as an associated database for larger aggregates of ice crystals (Nowell et al. 2013). Assumptions made with regard to ice particles can greatly impact modeled $T_{b}$ (Kulie et al. 2010). While we believe the assumptions made in our algorithm strike a reasonable balance between simplicity and accuracy, we acknowledge the substantial uncertainties involved. We direct the reader to SK19 for a quantification of the uncertainties and biases created by these forward model assumptions (see in particular Figs. 1 and 2).

\section{b. Construction of covariance matrices and forward model offsets}

The 1DVAR retrieval solution can be very sensitive to the error covariance matrices $\mathbf{S}_{a}$ and $\mathbf{S}_{y}$ (Duncan and Kummerow 2016), so it is important that the assumed uncertainties specified in these matrices are carefully calculated and not just ad hoc guesses. $\mathbf{S}_{a}$ uncertainties are estimated from covariances between state vector values in ERA5 reanalysis data, following SK19. More interesting is the calculation of the $\mathbf{S}_{y}$ matrix, as well as forward model $T_{b}$ offsets that are meant to account for systematic biases in the forward model and/or the PMW radiometer.

We start with TEMPEST-D-observed $T_{b}$ from 8 to 14 December 2018. We match ERA5 atmospheric profiles, at their full native vertical resolution, to the TEMPEST-D pixels, and calculate simulated TEMPEST-D $T_{b}$ using the same radiative transfer model used in the retrieval forward model. Pixels for which ERA5 indicates precipitation are excluded. Next, we create a second set of simulated observations; however, this time we reduce the accuracy of the simulated $T_{b}$ by making the same assumptions made in the retrieval algorithm. The vertical resolution is reduced, all of the cloud water and ice is constrained to lie within the levels specified in the retrieval (given above), and the vertical profiles of water vapor are simplified to that which can be best described by only three principal components (see SK19 for details on how the forward model handles the water vapor profile). We also add 

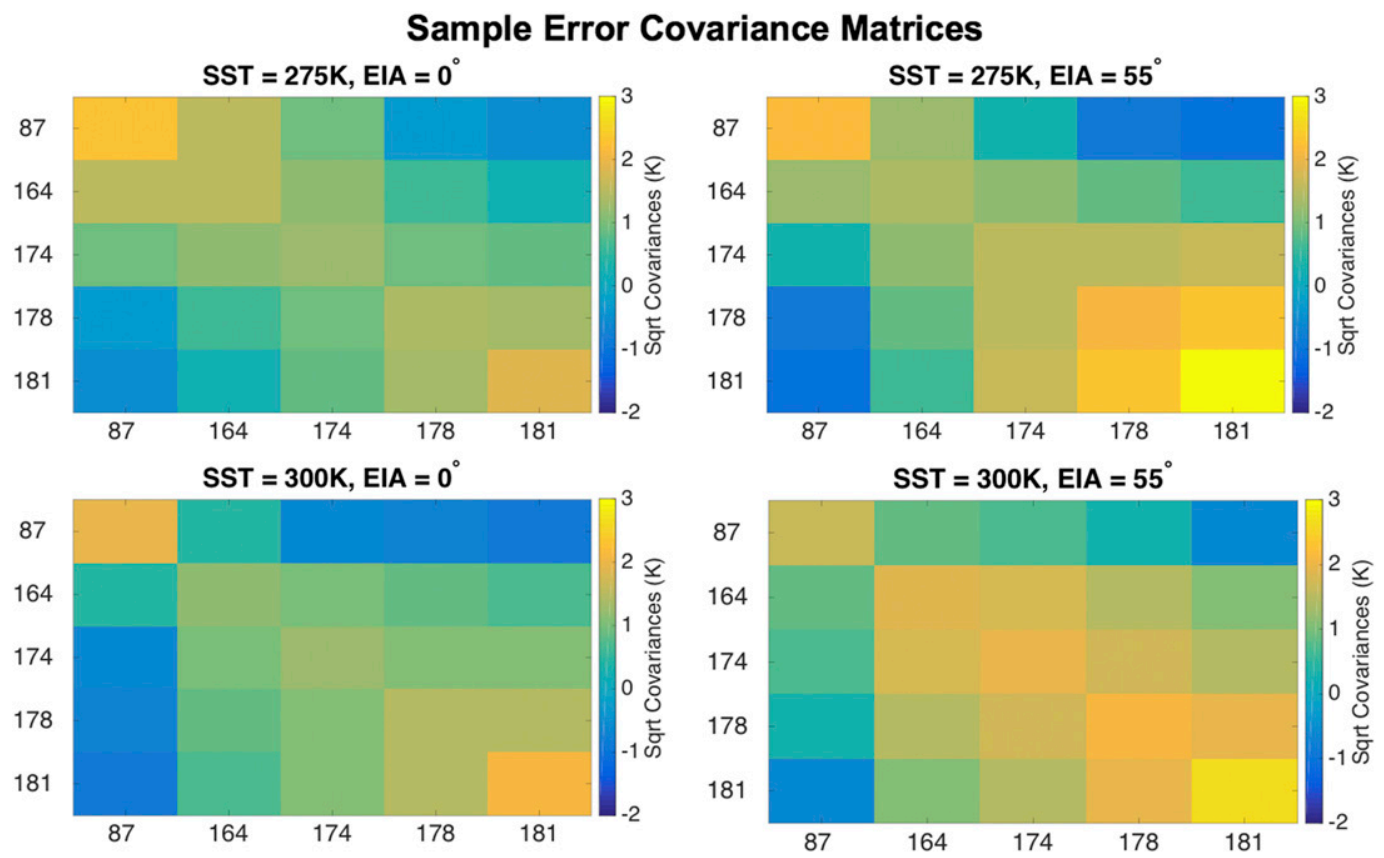

FIG. 2. Sample forward model error covariance matrices $\mathbf{S}_{y}$, for the SST bins 275-276 and 300-301 K and EIA bins $0^{\circ}-4^{\circ}$ and $52^{\circ}-56^{\circ}$. Values are given as the square root of the covariances so as to have units of kelvin. Negative covariances are shown as -1 times the square root of the absolute value of the covariance. The square root covariances are shown for the five radiometer channels centered at 87, 164, 174, 178 and $181 \mathrm{GHz}$.

random perturbations to the surface temperature and wind speed, the salinity, and the temperature profile, to mimic the real-world uncertainty present in the values used for the forward model's ancillary and assumed parameters.

By comparing these two sets of simulated $T_{b}$, one from a detailed representation of the atmosphere and the other from the simplified representation of the retrieval forward model, we can estimate the channel uncertainties related to the forward model. The $\mathbf{S}_{y}$ matrix is formed by calculating the covariances of the simulated minus simulated $T_{b}$ differences and then adding the TEMPEST-D NEDT for each channel to the diagonal elements of the matrix. In doing so we assume that instrument channel errors are uncorrelated, though it is true that some types of instrument errors, such as the error in specifying the hot-load temperature, are correlated across channels.

Each TEMPEST-D pixel without precipitation is binned based on the sea surface temperature for the pixel (SST) and the Earth incidence angle (EIA) between the radiometer boresight and the local vertical at the location of the pixel. We use 33 SST bins (in 1-K increments from 273 to $305 \mathrm{~K}$ ) and 30 angle bins (in $4^{\circ}$ increments from $-60^{\circ}$ to $60^{\circ}$ ). Then a separate $\mathbf{S}_{y}$ matrix is calculated for each bin, following SK19. Our justification for this is that the nature of the forward model errors is dependent both on the atmospheric regime (for which we use SST as a proxy) and on the view angle being considered. For example, colder SSTs are generally associated with reduced total column water vapor. The TEMPEST-D $87-\mathrm{GHz}$ channel is more sensitive to the surface when there is less water vapor, making that channel's uncertainties more sensitive to SST, wind speed, and emissivity model errors and thus increasing the channel uncertainty. This can be seen in Fig. 2. Likewise, when the EIA is large the slant path through the atmosphere will be longer and there will be less sensitivity to uncertainties in surface parameters. A larger EIA also corresponds to a larger instantaneous field of view, which can further change the nature of forward model errors due to field-of-view inhomogeneities. For the higher-frequency channels, the general trend is that, the more sensitive the channel is to the upper troposphere, the larger the uncertainty. Thus the uncertainty increases both as one moves closer in frequency to the $183-\mathrm{GHz}$ water vapor absorption line and as the EIA increases. The increased uncertainty is likely due to both the relatively coarse resolution of the upper troposphere in the forward model as well as the simplistic way in which ice clouds are represented. SK19 found that accounting for these EIA- and SST-dependent changes in the $\mathbf{S}_{y}$ matrix, when applying the CSU 1DVAR retrieval to MHS 
observations, made a small but significant difference in the final retrieved TPW values and led to a greater consistency across the MHS scans.

This procedure of estimating $\mathbf{S}_{y}$ accounts for many of the most significant sources of forward model error, particularly for clear sky conditions where scattering is not a factor. It also accounts for the random component of instrument error, through the addition of the channel NEDT. However, it does not account for systematic biases that might exist between the observed and forward modeled $T_{b}$. These biases could exist for many reasons and could be related to either instrument or forward model errors. For example, errors in the measurement of the calibration load temperature, or the intrusion of microwave radiation from a non-Earth source such as the spacecraft itself, could lead to an instrument bias. On the other hand, all of the sources of forward model error considered in the $\mathbf{S}_{y}$ calculation, as well as harder-to-quantify uncertainties such as absorption model and emissivity model errors, could contribute to a forward model bias. While disentangling all of these effects is difficult, estimating their cumulative impact is somewhat easier, and we correct for them by applying $T_{b}$ offsets, or bias corrections, to the TEMPEST-D observations before processing them.

To calculate these $T_{b}$ offsets, we compare the observed TEMPEST-D $T_{b}$ from 8 to 14 December 2018 with the set of $T_{b}$ simulated from ERA5 using the simplified model of the atmosphere. As mentioned above, the nature of forward model errors for each channel is somewhat dependent on the EIA and the SST regime being considered. This is true not only for the magnitude of the error variances and covariances (i.e., the information in $\mathbf{S}_{y}$ ) but also for systematic biases. Likewise, it is reasonable to suspect that instrument biases might also be dependent on scan position (John et al. 2013) or scene temperature (which will to some degree be correlated with SST). Thus, we bin the bias corrections by EIA and SST, just as we do the $\mathbf{S}_{y}$ covariances. For each SST/EIA combination bin, we calculate the median observed minus simulated $T_{b}$ from the observation period. We use the median rather than the mean because the median is less sensitive to outlier values that can result, for example, if ERA5 misplaces a frontal system relative to where the $T_{b}$ signature suggests it should be. The median values are then smoothed in EIA-SST space using a Gaussian convolution filter, and it is these smoothed values that are used as forward model offsets in the CSU 1DVAR retrieval. That is, the appropriate offset for a given pixel's EIA and SST is added to the output of the forward model before comparing it with the observed $T_{b}$. The forward model offsets for each TEMPEST-D channel are shown in Fig. 3, and it is clear that the offsets are complicated functions of both EIA and SST. This method of calculating offsets does risk incorporating biases that might be present in ERA5 into the final parameters retrieved from TEMPEST-D $T_{b}$ by the CSU 1DVAR algorithm. However, since possible ERA5 biases will be location dependent rather than EIA dependent, this does not deter our primary objective of evaluating the consistency of TEMPEST-D retrievals as a function of EIA.

\section{Results}

\section{a. Consistency with MHS}

The MHS radiometer, with a set of channels that are quite similar to TEMPEST-D, is a natural instrument against which to compare observations. One way to assess the quality of TEMPEST-D observations is to compare TEMPEST-D $T_{b}$ to MHS $T_{b}$ using the "double difference method." This method shows that TEMPEST-D $T_{b}$ are consistent with MHS $T_{b}$ to within about $1 \mathrm{~K}$ (slightly larger differences exist at the $164-\mathrm{GHz}$ channel, because of band mismatches and surface emissivity sensitivity), and that the calibration differences are stable with time (Berg et al. 2019).

Another way to evaluate the consistency with MHS is to look at retrieved products. MHS instruments are in sunsynchronous polar orbits, which means that the field of view of TEMPEST-D coincides with the field of view of each MHS radiometer two times per orbit. Figure 4 shows an example of such an overpass from 9 December 2018. In this case, TEMPEST-D made observations over the western Pacific Ocean around 1124 UTC that were nearly coincident with observations from the MetOp-B satellite. Figure 4 compares the TPW, LWP, and IWP retrieved from TEMPEST-D by the CSU 1DVAR algorithm to that retrieved from $M e t O p-B$ by the MiRS algorithm. The top plots show the TEMPEST-D values with the MiRS swath in the background, and in the bottom plots the order is reversed. The two products are in broad agreement. They agree quite well on the placement of liquid phase clouds to the south of Japan, for instance, as well as the existence of ice particles north of Papua New Guinea. The main features of the water vapor field are the same, and there are no sharp gradients in TPW when the two retrieved swaths are plotted on top of each other.

Looking at all TEMPEST-D/MHS near-coincident observations from the 8-14 December period, the story is much the same. To identify all such observations, the data were gridded on an Earth-fixed $0.25^{\circ}$ grid (necessary because the MHS and TEMPEST-D footprints and ground tracks do not match), and observations taken within $5 \mathrm{~min}$ of each other were included for analysis. This resulted in 17869 instances of matched pixels that were over the ocean and had valid data for both TEMPEST-D and MiRS. Summary statistics for the difference in 


\section{Forward Model Offsets}
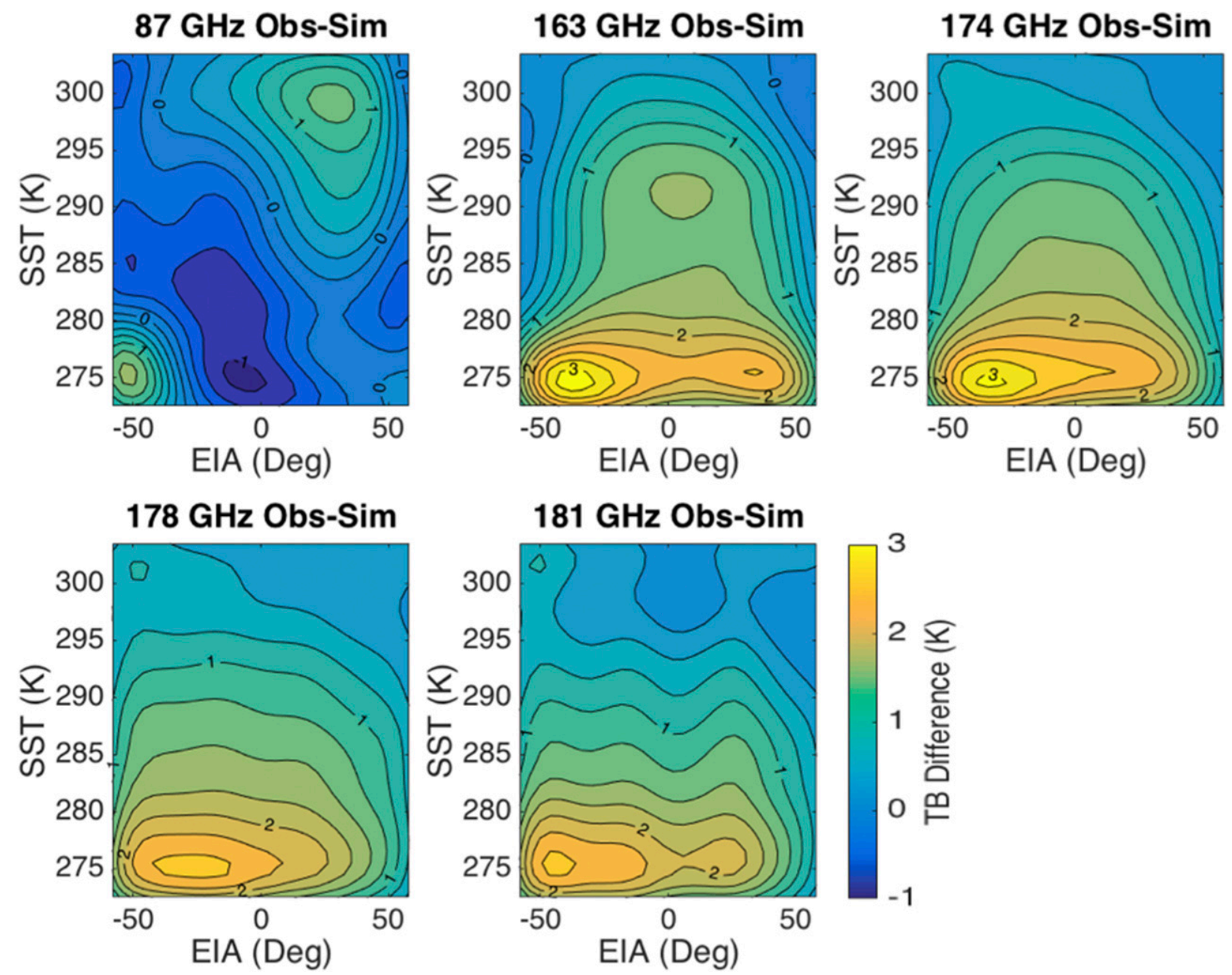

FIG. 3. Median TEMPEST-D observed $T_{b}$ minus forward model simulated $T_{b}$ from ERA5, for all TEMPEST-D orbits from 8 to 14 Dec 2018 and as a function of EIA and SST. The contour lines are plotted in increments of $0.25 \mathrm{~K}$. These offsets are applied to simulated $T_{b}$ in the retrieval algorithm before the simulated $T_{b}$ are compared with the observed ones.

retrieved TPW and LWP are given in Table 2, and scatterplots between TEMPEST-D and MiRS values are shown in Figs. 5 and 6 for TPW and LWP, respectively. Because of the time period considered and the inclination of the TEMPEST-D and MHS orbits, most of the nearcoincident observations occurred in the midlatitudes, so we caution that the relationships presented here might be different in other regimes.

Retrieved TPW is correlated very highly, with a correlation coefficient of 0.976 and a standard deviation of the difference between the two values of $2.87 \mathrm{~mm}$. TEMPEST-D TPW is biased low (negatively) relative to MiRS TPW; however, note that SK19 found that MiRS TPW estimates were biased high (positively) relative to ground-based SuomiNet estimates, so this puts TEMPEST-D estimates more in line with SuomiNet. The correlation between LWP estimates is not as strong ( $r=0.692)$, as evident in Fig. 6, but this is to be expected. LWP is inherently harder to retrieve (e.g., it is very hard to radiometrically distinguish cloud water from rainwater) and can vary dramatically on small spatial scales. Even if both the MiRS and TEMPEST-D retrieval algorithms were perfect, one would expect to see considerable differences in retrieved LWP for pixels up to $0.25^{\circ}$ apart in space and up to $5 \mathrm{~min}$ apart in time.

\section{b. Consistency across scan}

In SK19, it was shown that MiRS TPW estimates from MHS instruments tended to be higher near nadir and drop off at large view angles, and that a similar pattern 


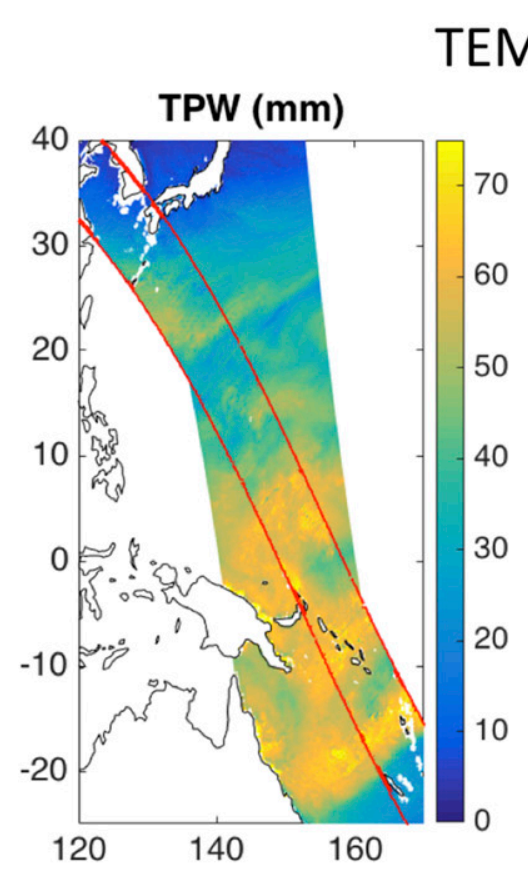

\section{TEMPEST-D Plotted Over MiRS}

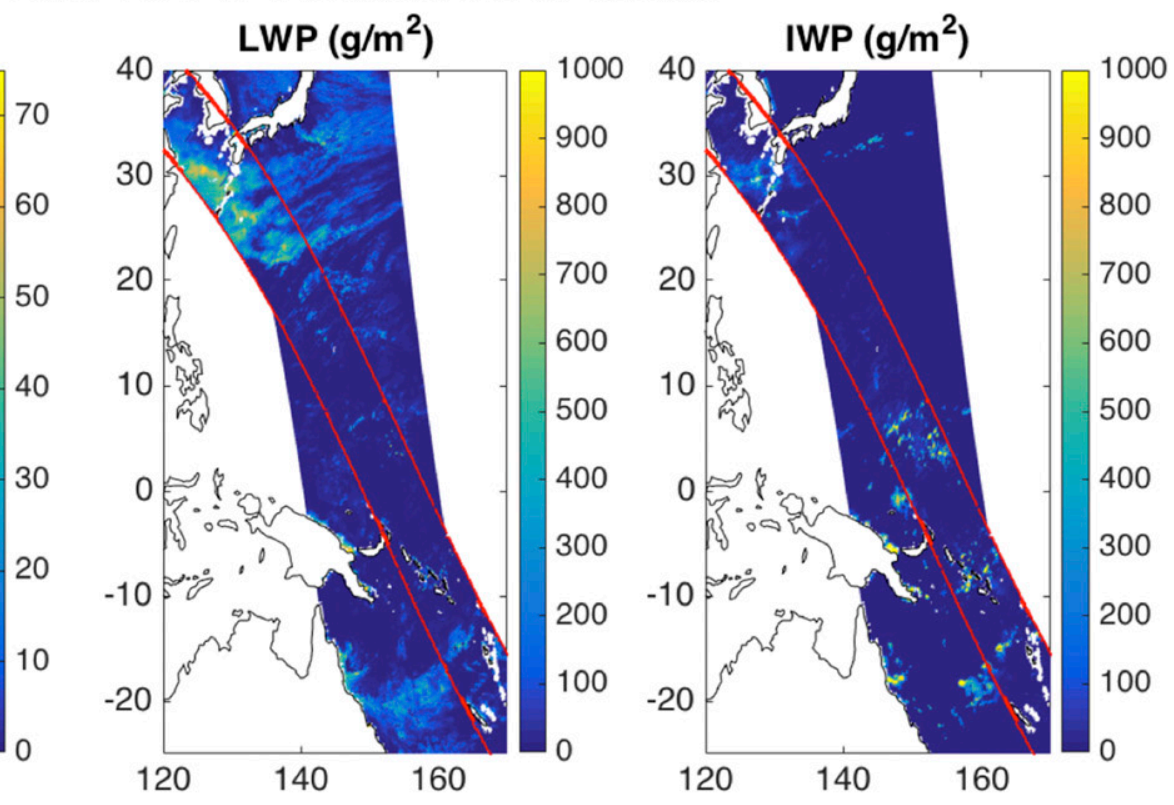

\section{MiRS Plotted Over TEMPEST-D}
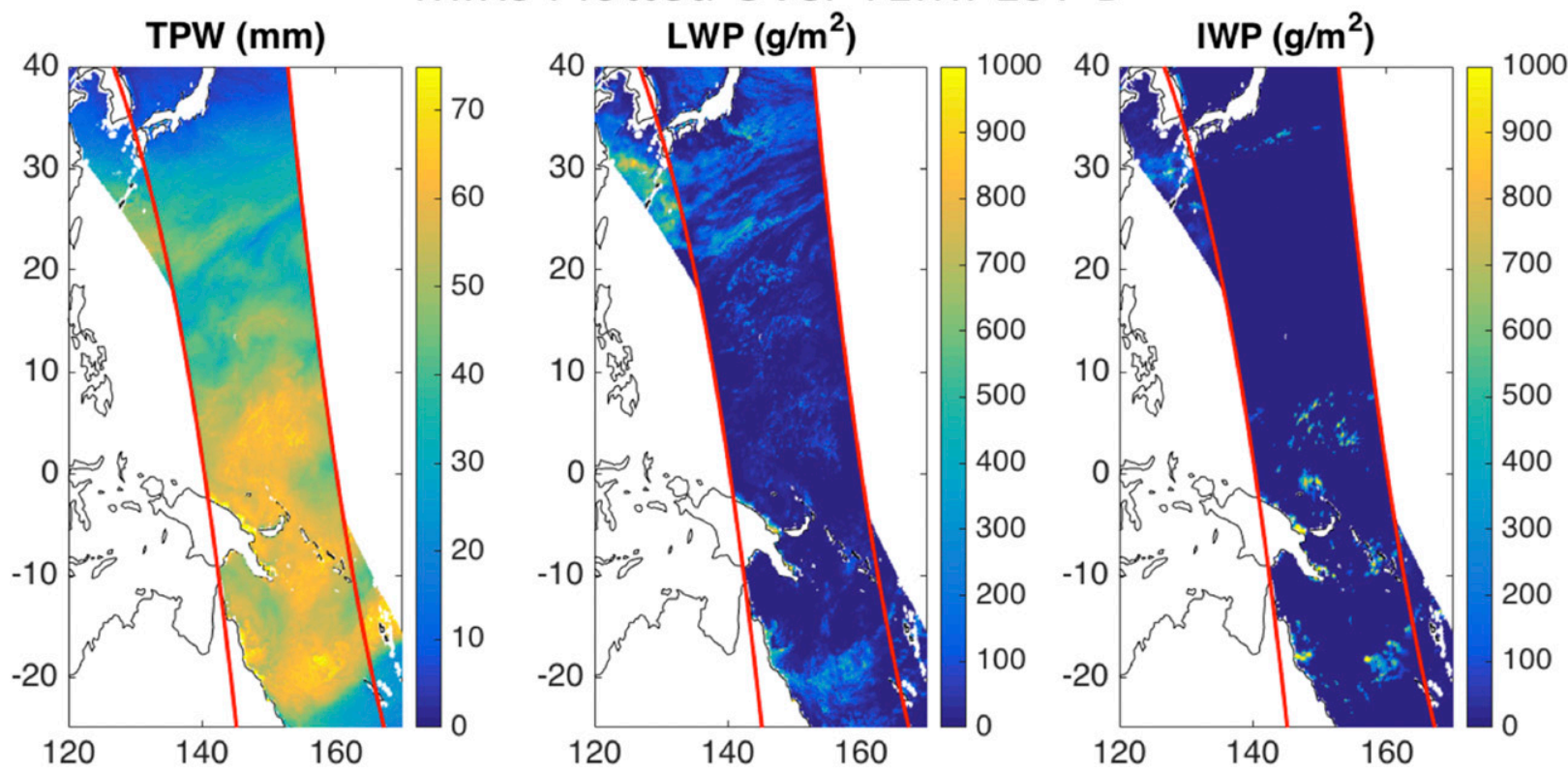

FIG. 4. TPW, LWP, and IWP retrieved from the TEMPEST-D and Met $O p-B$ satellites for a coincident overpass near 1124 UTC 9 Dec 2018: (top) the retrieved TEMPEST-D fields from the CSU 1DVAR retrieval algorithm plotted on top of the $M e t O p$ - $B$ retrieved values and (bottom) the $M e t O p-B$ values from MiRS plotted on top of the retrieved TEMPEST-D fields.

was seen in CSU 1DVAR TPW estimates from crosstrack instruments when constant (i.e., no SST or EIA dependence) error covariance assumptions were used. Since MiRS employs scan position-based $T_{b}$ offsets meant to account for instrument errors, and the CSU 1DVAR was run using intercalibrated MHS $T_{b}$ that should theoretically have no scan asymmetry, it was speculated that this pattern might be the result of shared forward model errors (such as the algorithms' use of the same surface emissivity model). When the CSU 1DVAR algorithm was rerun using a variable $\mathbf{S}_{y}$ matrix and forward model offsets that changed based on EIA and SST, this across-scan bias was almost totally eliminated, even when the algorithm was run on data from different time 
TABLE 2. Error statistics for TEMPEST-D retrieved values from 8 to 14 Dec 2018 compared with near-coincident MiRS values from the MetOp-A, MetOp-B, and NOAA-19 satellites. Bias values are TEMPEST-D minus MiRS.

\begin{tabular}{lcc}
\hline \hline & TPW & LWP \\
\hline Correlation coef & 0.976 & 0.692 \\
Bias & $-1.63 \mathrm{~mm}$ & $-0.72 \mathrm{~g} \mathrm{~m}^{-2}$ \\
Std dev & $2.87 \mathrm{~mm}$ & $49.01 \mathrm{~g} \mathrm{~m}^{-2}$ \\
\hline
\end{tabular}

periods and from satellites other than the one used to calculate the forward model adjustments. This supported the hypothesis that forward model errors were mostly responsible for the asymmetry, and that view-anglerelated biases could be corrected by accounting for these forward model errors (even if the specific causes of the forward model errors remained hard to diagnose).

Here we perform similar experiments using the TEMPEST-D instrument, and we once again find that the methodology presented in section $3 \mathrm{~b}$ is able to largely mitigate view-angle-related biases. One way to address this question is to compare TEMPEST-D retrieved TPW as a function of EIA with reanalysis data. We use the European Centre for Medium-Range Weather Forecasts' reanalysis product, ERA5 (ECMWF 2017), for this purpose. Considering that ERA5 incorporates a physically based atmospheric model, and that TEMPEST-D observations are not assimilated into ERA5, ERA5 TPW errors should be independent of TEMPEST-D view angle. Thus, when comparing a retrieved product to ERA5, one would expect to find a nearly constant average difference with respect to EIA. Figure 7 shows the result of this sort of comparison for all TEMPEST-D pixels from 8 to 14 December, and confirms

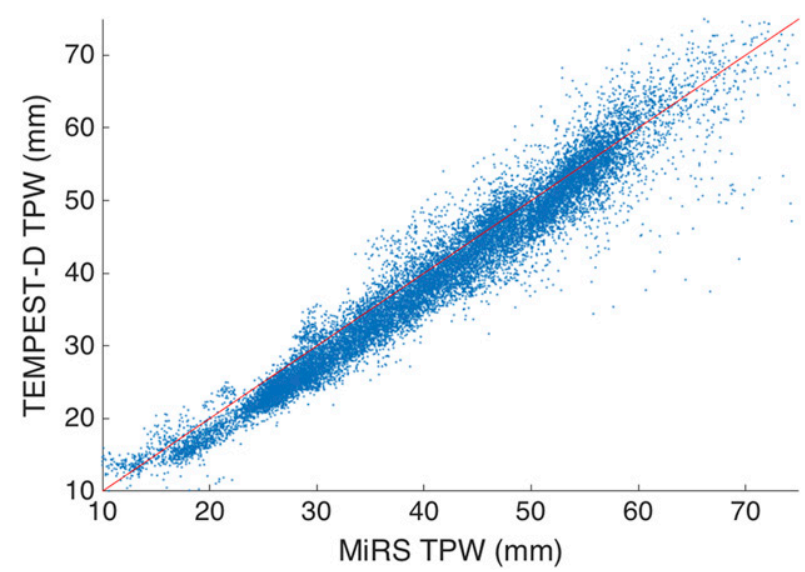

FIG. 5. Scatterplot comparing MiRS TPW from the MHS instruments on MetOp-A, MetOp-B, and NOAA-19 with TPW retrieved from TEMPEST-D, for all coincident observations $(n=17869)$ from 8 to 14 Dec 2018 . Data have been gridded with a $0.25^{\circ}$ resolution, and observations are counted as coincident if they occur at the same grid point within $5 \mathrm{~min}$ of each other. The red line is the one-to-one line.

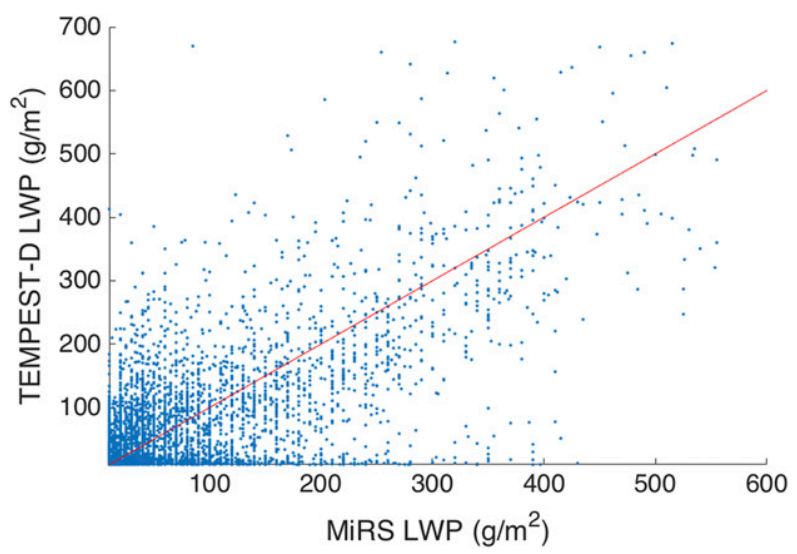

FIG. 6. As in Fig. 5, but for LWP.

that the difference with respect to EIA is nearly flat. Also shown for comparison is the average (MetOp-B) MiRS TPW bias relative to ERA5 as a function of EIA, and the same edge-of-scan roll-off found in SK19 is evident.

However, with the unique yaw maneuver dataset from TEMPEST-D, it is possible to examine potential viewangle-related biases more directly than in SK19. While the satellite was in the along-track scanning mode, the TEMPEST-D instrument viewed the same locations many times in quick succession. These views can be categorized according to EIA to examine the consistency of retrieved products in a much more direct way than was possible in SK19. Figure 8 shows an example of this. A TEMPEST-D nadir-viewing pixel (located at the spot marked " $X$ " in Fig. 9) is taken as the reference point and all preceding or subsequent observations whose center field-of-view point is within $10 \mathrm{~km}$ of the center of the reference pixel are considered to be coincident. Figure 8 shows that the retrieved TPW and LWP (IWP is negligible in this case) are quite consistent for all retrievals, with no noticeable dependence on EIA.

This particular case study was chosen in part because it occurred simultaneous with a $\mathrm{MetO} \mathrm{O}-\mathrm{B}$ overpass with MHS observations. The CSU 1DVAR algorithm was also run on the MHS pixel closest to the reference point for comparison. This value (which was associated with an EIA of approximately $27^{\circ}$ ) is shown by the constant red line in Fig. 8. One of the advantages of the optimal estimation framework is that explicit error estimates are provided by the posterior error covariance matrix. The red dashed lines in Fig. 8 represent the \pm 1 standard deviation uncertainty ranges for the MHS-based estimates. The first thing to note is that the TEMPEST-D estimates agree reasonably well with the MHS estimates. This is especially true considering that the TEMPEST-D and MHS footprints do not align perfectly, and that the observations were taken a few minutes apart from each 


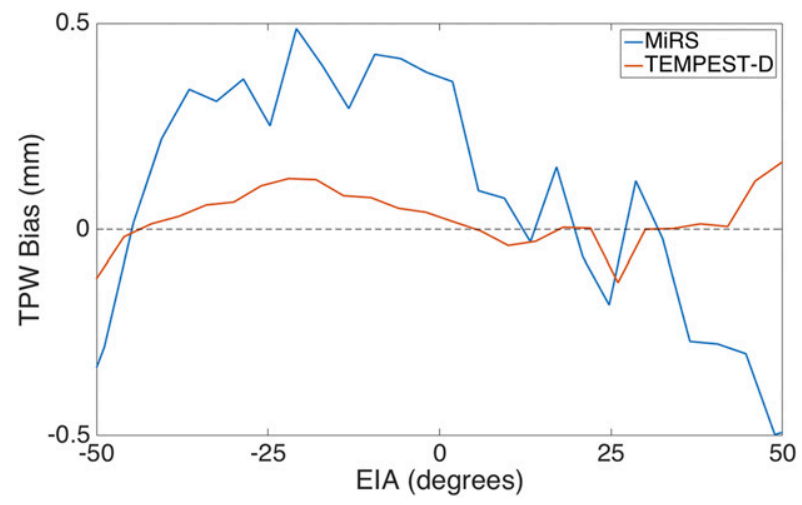

FIG. 7. Mean difference between retrieved TPW and ERA5 reanalysis TPW as a function of EIA for the period 8-14 Dec 2018 from the MiRS retrieval run on MetOp-B satellite data (blue) and from the CSU 1DVAR retrieval run on TEMPEST-D satellite data (red). The overall mean bias between each retrieval and ERA5 has been removed.

other. Additionally, the variation between the different TEMPEST-D estimates is smaller than the uncertainty associated with the single MHS estimate. This suggests that the retrieval uncertainty is driven more by uncertain forward model assumptions (which are common to all observations) than by instrument uncertainties or view-angle differences.

Taking a larger view, Fig. 9 shows the full context in which this comparison was made. The TEMPEST-D ground track is plotted on top of the coincident MHS swath, with CSU 1DVAR retrieved values of TPW and LWP. TEMPEST-D crosses a sharp water vapor gradient near $50^{\circ} \mathrm{S}, 30^{\circ} \mathrm{E}$ and also passes over two significant cloud clusters. The middle panels in Fig. 9 show all TEMPEST-D pixels located within $10 \mathrm{~km}$ of the red ground track, categorized by EIA and longitude and colored according to the retrieved value of TPW or LWP. Matching the colors in these panels to the corresponding locations in the top panels, it is seen that there is good agreement between the TEMPEST-D and MHS observations, particularly with regard to the sharp water vapor gradient and the location of clouds. The vertical "stripes" in these plots show that the retrieved values at a given location tend to be very consistent as a function of EIA. The consistency with MHS retrievals and between retrievals taken at different view angles is also apparent when looking at the bottom panels, which plot the spread of TEMPEST-D values retrieved along the ground track at all view angles compared with MHS retrieved values for the MHS pixels closest to the ground track. From these plots one can see that the TEMPEST-D retrievals mostly fall within the MHS error bounds, and also that the spread of the TEMPEST-D retrievals is smaller than the overall uncertainty in the MHS retrievals.
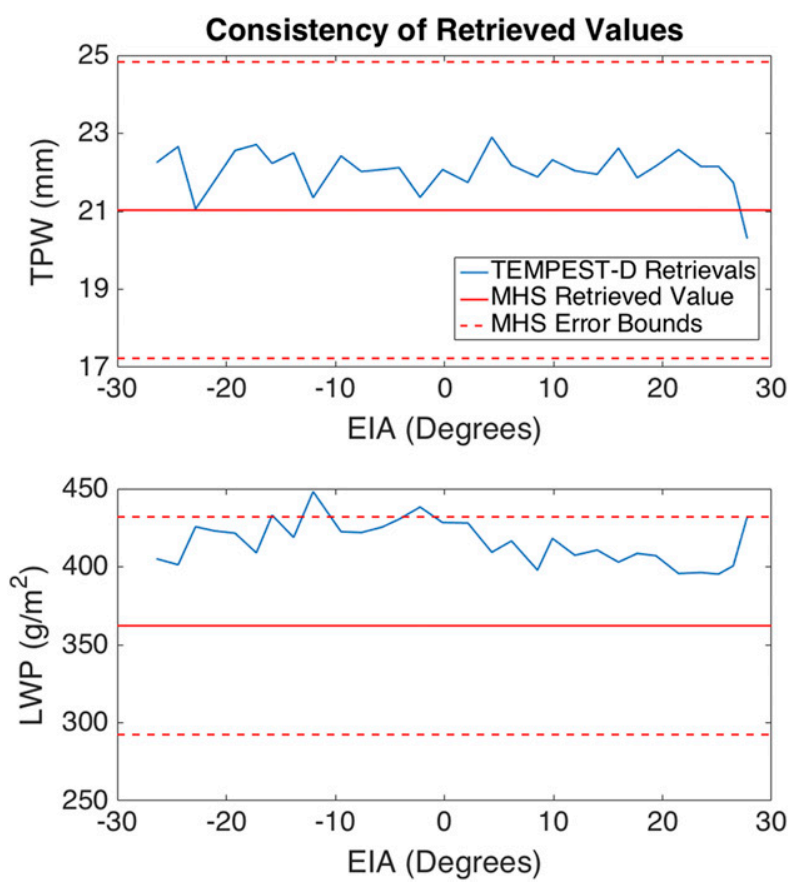

FIG. 8. The blue line shows all TEMPEST-D retrieved values of (top) TPW and (bottom) LWP from 30 Jan 2019, near 0600 UTC and within $10 \mathrm{~km}$ of the point $50.17^{\circ} \mathrm{S}, 33.25^{\circ} \mathrm{E}$, as a function of EIA. The solid red line represents the corresponding single value retrieved from a near-coincident observation by MHS, with the red dashed lines representing \pm 1 standard deviation, as reported by the posterior covariance matrix.

We also consider the entirety (all $73 \mathrm{~h}$ ) of the TEMPEST-D yaw maneuver dataset. Observations are binned into $4^{\circ}$ bins according to their EIA, and the retrieved TPW and LWP are compared with those retrieved by TEMPEST-D at the same point at nadir (if no such observation exists within $5 \mathrm{~km}$ of the pixel being considered, that pixel is excluded from the analysis). Figure 10 shows the median difference between these observations and observations of the same location at nadir. The median difference is nearly independent of EIA for TPW, and while the relationship for LWP is slightly noisier, the relationship with EIA is also largely flat.

Figure 10 also shows the resulting biases when the CSU 1DVAR algorithm is run on the TEMPEST-D yaw maneuver data without variable forward model $T_{b}$ offsets or error covariance matrices. The mean $T_{b}$ offset and average $\mathbf{S}_{y}$ matrix, across all EIA and SST bins, are used instead. In this case, there are clear patterns in the TPW and LWP biases with respect to EIA. On the TPW side, there is both the familiar edge-of-scan roll-off that is seen in the MiRS data as well as a left-to-right asymmetry. This is probably the result of an instrument asymmetry, as a similar pattern is seen when comparing TEMPEST-D 87-GHz $T_{b}$ to collocated MHS $89-\mathrm{GHz}$ 

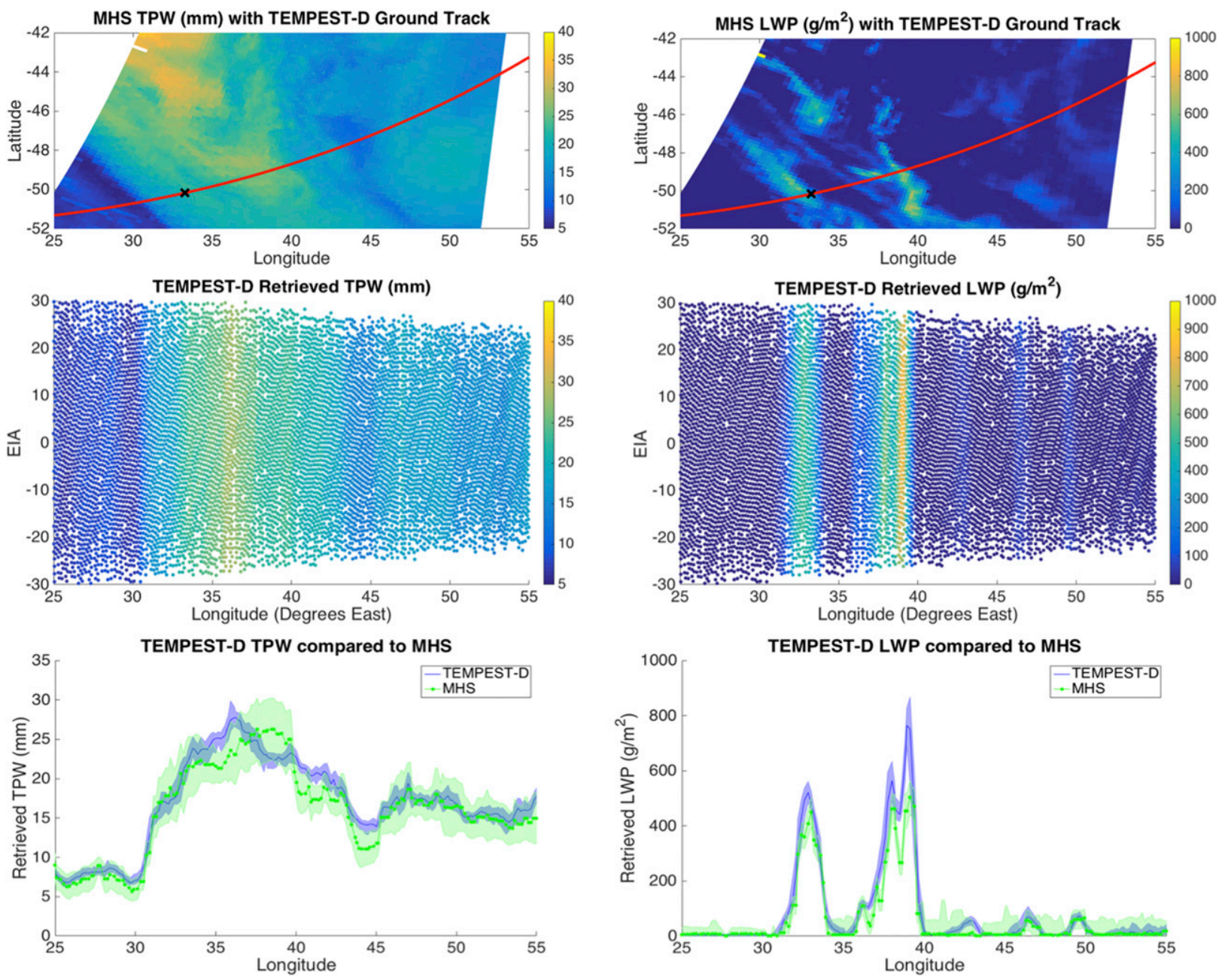

FIG. 9. (top left) TPW and (top right) LWP retrieved by the CSU 1DVAR algorithm for an MHS overpass from $M e t O p$ - $B$ over the Southern Ocean on 30 Jan 2019 around 0600 UTC. The red line shows the ground track of a coincident TEMPEST-D overpass while the TEMPEST-D satellite was in along-track scanning mode. The black X shows the location of the MHS pixel used as a comparison point in Fig. 8. (middle) All of the TEMPEST-D pixels within $10 \mathrm{~km}$ of the TEMPEST-D ground track, plotted with respect to longitude and EIA. The color of each dot represents the magnitude of (left) TPW or (right) LWP retrieved. (bottom left) TPW and (bottom right) LWP (solid blue lines) retrieved by TEMPEST-D at nadir along the ground track, with the shading showing the full range of values retrieved for the corresponding pixel at all view angles. The green line is the value retrieved at the closest MHS pixel, with shading representing \pm 1 standard deviation, as reported by the posterior covariance matrix.

$T_{b}$, and the TEMPEST-D $T_{b}$ have not been calibrated in any way except for the process of calculating forward model offsets. Meanwhile, the LWP plot shows that, under this scenario, LWP becomes biased high at high view angles, perhaps to compensate for missing TPW. A third experiment was run in which $\mathbf{S}_{y}$ matrices and forward model offsets were binned by EIA, but not by SST. This is similar to the current setup of MiRS. In this case, the edge-of-scan bias is reduced but not eliminated. This demonstrates that, because the nature of EIA-dependent forward model errors is different for different atmospheric regimes, nuanced error assumptions that take into account both EIA and atmospheric conditions are necessary to fully eliminate view-anglerelated biases. In a final experiment, the forward model offsets were allowed to vary based upon both EIA and SST, but the $\mathbf{S}_{y}$ matrix was held constant. Consistent with SK19, the variable forward model offsets are primarily responsible for improving the across-scan consistency; however, the variable $\mathbf{S}_{y}$ matrix does play a small role as well.

\section{Conclusions}

TEMPEST-D observations show that CubeSat missions offer the potential to greatly increase the frequency 

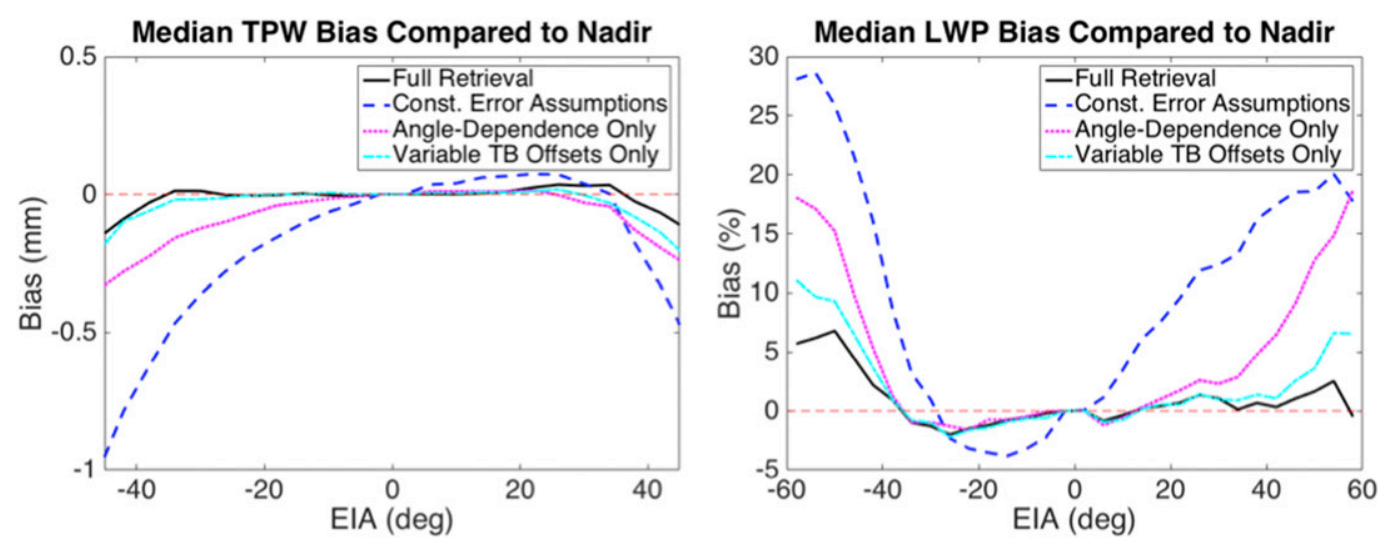

FIG. 10. (left) Median difference between TPW retrieved by TEMPEST-D at a given location and the TPW retrieved at the same location when the instrument was looking at nadir, for all yaw maneuver observations in the data record with latitudes between $45^{\circ} \mathrm{S}$ and $45^{\circ} \mathrm{N}$. Observations are considered to be collocated if the centers of their respective instantaneous fields of view are within $5 \mathrm{~km}$ of each other. Results are shown for the full retrieval (with both forward model offsets and an $\mathbf{S}_{y}$ matrix that depend on EIA and SST), for the retrieval with no EIA- or SST-dependent error assumptions at all, for the retrieval with EIA (but not SST) dependent $\mathbf{S}_{y}$ and offsets, and for the retrieval with EIA and SST dependent offsets but constant $\mathbf{S}_{y}$. (right) The same type of plot, but for LWP. The $y$ axis is in terms of percentage difference because of the wide range of values possible for LWP.

of PMW observations across the globe, for use in forecasting, data assimilation, and process studies. TEMPESTD measurements appear to be of similar quality to MHS measurements, as evidenced both by the consistency between TEMPEST-D and MHS $T_{b}$ and (as demonstrated in this study), the consistency in atmospheric parameters retrieved from TEMPEST-D by the CSU 1DVAR algorithm and those retrieved from MHS instruments. This is true both of MiRS retrievals and of CSU 1DVAR retrievals performed on MHS data.

One important consideration when it comes to crosstrack scanning PMW instruments like TEMPEST-D or MHS is the potential for across-scan biases in retrieved parameters. This becomes critical as the time sampling of PMW observations increases to explore changes in an atmospheric parameter that occur between observations. In order for actual changes from one observation to another (made with a different satellite and likely with a different viewing geometry) to be detected, one must have confidence that differences in retrieved values are due to actual physical changes and not due to forward model errors that depend on view angle.

The CSU 1DVAR algorithm has been shown to have near-zero view-angle bias when it comes to the retrieval of TPW and LWP from TEMPEST-D observations. This is true both when comparing TEMPEST-D retrieved values to reanalysis estimates and when looking directly at the same location many times with the TEMPEST-D instrument when the satellite was performing yaw maneuvers. The elimination of view-angledependent errors is achieved only when assumptions about both systematic and random errors are allowed to change based on SST regime and instrument EIA. Systematic errors are accounted for in the forward model $T_{b}$ offsets while random errors are specified in the error covariance matrix $\mathbf{S}_{y}$. The physical justification for doing this is that the nature of forward model errors will change with atmospheric regime and EIA, as demonstrated in SK19. When constant error assumptions are used instead, a clear EIA-related pattern is seen in the TPW and LWP biases. A similar TPW pattern is seen in MiRS retrievals on MHS data, suggesting that there might be forward model errors that are common to both algorithms. Other optimal-estimation-based retrieval algorithms might benefit from adopting the approach presented here of varying error assumptions based on SST and EIA. While we have used SST (which is correlated with TPW) as a proxy for atmospheric regime, other methods including atmospheric air mass could be explored.

The lessons learned through the development of the CSU 1DVAR algorithm about view-angle-related biases for cross-track scanning PMW radiometers could be useful for the upcoming TROPICS mission (Blackwell et al. 2018). TROPICS will consist of six CubeSats with PMW radiometers measuring at similar frequencies to TEMPEST-D (with the addition of several channels near the $118.75-\mathrm{GHz}$ oxygen absorption line) that will be launched into three different orbital planes, providing rapid-refresh PMW measurements in the tropics. With refresh times under one hour in some cases, it will be important to consider the impact different view 
angles could have on observations and to mitigate viewangle biases as much as possible. This work could also be of interest to the data assimilation community, since it is possible that the radiative transfer models used to assimilate PMW satellite observations could have similar view-angle-dependent errors to the errors present in the CSU 1DVAR forward model.

We acknowledge that one limitation of the study is that the same time period used to calculate the forward model offsets and error covariance matrix is also used to test the retrieval algorithm against MiRS. However, good retrieval results are also seen during the January and April along-track scanning periods, using the same offsets and covariances. In addition, the concentration of TEMPEST-D/MHS overpasses in the midlatitudes on the dates studied precludes a more thorough analysis of possible regional biases. As more TEMPEST-D data are collected, it will become possible to conduct even more rigorous statistical analyses and explore seasonal and regional dependencies.

Last, we note that the TEMPEST-D yaw maneuver data used in this study offer many possible avenues for further exploration. The TEMPEST-D along-track observations from clear-sky areas might be able to yield some insight into possible angle-dependent surface emissivity model errors near 87 and $164 \mathrm{GHz}$. In addition, looking at the same scenes from multiple angles gives additional information that could be used to investigate the vertical structure of water vapor and clouds. The CSU IDVAR algorithm framework is flexible enough that it could be modified to include multiangle observations. The TEMPEST-D data are publicly available online (https:// tempest.colostate.edu/data).

Acknowledgments. The authors thank Dr. David Duncan for numerous helpful discussions throughout the development of the CSU 1DVAR algorithm and thank the entire TEMPEST-D team at Colorado State University, the California Institute of Technology Jet Propulsion Laboratory, and Blue Canyon Technologies for their work in building and launching TEMPEST-D. The work was supported by NASA Earth Venture Program Grant NNX15AP56G.

\section{REFERENCES}

Berg, W., and Coauthors, 2019: Demonstrating the viability of the TEMPEST-D CubeSat radiometer for science applications. 2019 IEEE Int. Geoscience and Remote Sensing Symp., Yokohama, Japan, IEEE, 8426-8428, https://doi.org/10.1109/ IGARSS.2019.8897881.

Blackwell, W. J., and Coauthors, 2018: An overview of the TROPICS NASA Earth venture mission. Quart. J. Roy. Meteor. Soc., 144 (Suppl. 1), 16-26, https://doi.org/10.1002/qj.3290.
Boukabara, S.-A., K. Garrett, and W. Chen, 2010: Global coverage of total precipitable water using a microwave variational algorithm. IEEE Trans. Geosci. Remote Sens., 48, 3608-3621, https://doi.org/10.1109/TGRS.2010.2048035.

__ , and Coauthors, 2011: MiRS: An all-weather 1DVAR satellite data assimilation and retrieval system. IEEE Trans. Geosci. Remote Sens., 49, 3249-3272, https://doi.org/ 10.1109/TGRS.2011.2158438.

Clough, S. A., M. W. Shephard, E. J. Mlawer, J. S. Delamere, M. J. Iacono, K. Cady-Pereira, S. Boukabara, and P. D. Brown, 2005: Atmospheric radiative transfer modeling: A summary of the AER codes. J. Quant. Spectrosc. Radiat. Transfer, 91, 233-244, https://doi.org/10.1016/j.jqsrt.2004.05.058.

Duncan, D. I., and C. D. Kummerow, 2016: A 1DVAR retrieval applied to GMI: Algorithm description, validation, and sensitivities. J. Geophys. Res. Atmos., 121, 7415-7429, https:// doi.org/10.1002/2016JD024808.

ECMWF, 2017: ERA5 Reanalysis. National Center for Atmospheric Research Computational and Information Systems Laboratory, accessed 1 June 2018, https://doi.org/10.5065/D6X34W69.

Field, P. R., A. J. Heymsfield, and A. Bansemer, 2007: Snow size distribution parameterization for midlatitude and tropical ice clouds. J. Atmos. Sci., 64, 4346-4365, https://doi.org/10.1175/ 2007JAS2344.1.

Geer, A. J., and Coauthors, 2017: The growing impact of satellite observations sensitive to humidity, cloud, and precipitation. Quart. J. Roy. Meteor. Soc., 143, 3189-3206, https://doi.org/ 10.1002/qj.3172.

Greenwald, T. J., G. L. Stephens, T. H. Vonder Haar, and D. L. Jackson, 1993: A physical retrieval of cloud liquid water over the global oceans using Special Sensor Microwave/Imager (SSM/I) observations. J. Geophys. Res., 98, 18471-18478, https://doi.org/10.1029/93JD00339.

John, V. O., G. Holl, N. Atkinson, and S. A. Buehler, 2013: Monitoring scan asymmetry of microwave humidity sounding channels using simultaneous all angle collocations (SAACs). J. Geophys. Res. Atmos., 118, 1536-1545, https://doi.org/ 10.1002/jgrd.50154.

Kazumori, M., and S. J. English, 2015: Use of the ocean surface wind direction signal in microwave radiance assimilation. Quart. J. Roy. Meteor. Soc., 141, 1354-1375, https://doi.org/10.1002/qj.2445.

Kulie, M. S., R. Bennartz, T. J. Greenwald, Y. Chen, and F. Weng, 2010: Uncertainties in microwave properties of frozen precipitation: Implications for remote sensing and data assimilation. J. Atmos. Sci., 67, 3471-3487, https://doi.org/10.1175/ 2010JAS3520.1.

Liu, G., 2008: A database of microwave single-scattering properties for nonspherical ice particles. Bull. Amer. Meteor. Soc., 89, 1563-1570, https://doi.org/10.1175/2008BAMS2486.1.

Ma, Y., Z. Xiaolei, and F. Weng, 2017: Potential applications of small satellite microwave observations for monitoring and predicting global fast-evolving weathers. IEEE J. Sel. Top. Appl. Earth Obs. Remote Sens., 10, 2441-2451, https://doi.org/ 10.1109/JSTARS.2017.2663335.

Molod, A., L. Takacs, M. Suarez, J. Bacmeister, I.-S. Song, and A. Eichmann, 2012: The GEOS-5 Atmospheric General Circulation Model: Mean climate and development from MERRA to Fortuna. Global Modeling and Data Assimilation, Vol. 28, NASA Tech. Rep. Series, NASA TM-2012-104606, $117 \mathrm{pp}$.

Nowell, H., G. Liu, and R. Honeyager, 2013: Modeling the singlescattering properties of aggregate snowflakes. J. Geophys. Res. Atmos., 118, 7873-7885, https://doi.org/10.1002/jgrd.50620. 
Reising, S. C., and Coauthors, 2018: An Earth venture in-space technology demonstration mission for Temporal Experiment for Storms and Tropical Systems (TEMPEST). 2018 IEEE Int. Geoscience and Remote Sensing Symp., Valencia, Spain, IEEE, 6301-6303, https://doi.org/10.1109/IGARSS.2018.8517330.

Rodgers, C. D., 2000: Inverse Methods for Atmospheric Sounding: Theory and Practice. World Science, 240 pp.

Schulte, R. M., and C. D. Kummerow, 2019: An optimal estimation retrieval algorithm for microwave humidity sounding channels with minimal scan position bias. J. Atmos. Oceanic Technol., 36, 409-425, https://doi.org/10.1175/JTECH-D-18-0133.1.

Wentz, F. J., 1997: A well-calibrated ocean algorithm for Special Sensor Microwave/Imager. J. Geophys. Res., 102, 8703-8718, https://doi.org/10.1029/96JC01751.

Wilheit, T. T., and A. T. C. Chang, 1980: An algorithm for retrieval of ocean's surface and atmospheric parameters from the observations of the scanning multichannel microwave radiometer. Radio Sci., 15, 525-544, https://doi.org/10.1029/RS015i003p00525. 